\title{
Coordination Strategy of Large-Scale DFIG-based Wind Farm for Voltage Support with High Converter Capacity Utilization
}

\author{
Document Version \\ Accepted author manuscript
}

Link to publication record in Manchester Research Explorer

Citation for published version (APA):

Dong, Z., Li, Z., Du, L., Liu, Y., \& Ding, Z. (Accepted/In press). Coordination Strategy of Large-Scale DFIG-based Wind Farm for Voltage Support with High Converter Capacity Utilization. IEEE Transactions on Sustainable Energy.

\section{Published in:}

IEEE Transactions on Sustainable Energy

\section{Citing this paper}

Please note that where the full-text provided on Manchester Research Explorer is the Author Accepted Manuscript or Proof version this may differ from the final Published version. If citing, it is advised that you check and use the publisher's definitive version.

\section{General rights}

Copyright and moral rights for the publications made accessible in the Research Explorer are retained by the authors and/or other copyright owners and it is a condition of accessing publications that users recognise and abide by the legal requirements associated with these rights.

\section{Takedown policy}

If you believe that this document breaches copyright please refer to the University of Manchester's Takedown Procedures [http://man.ac.uk/04Y6Bo] or contact uml.scholarlycommunications@manchester.ac.uk providing relevant details, so we can investigate your claim.

\section{OPEN ACCESS}




\title{
Coordination Strategy of Large-Scale DFIG-based Wind Farm for Voltage Support with High Converter Capacity Utilization
}

\author{
Zhen Dong, Zhongguo Li, Lingyu Du, Yixing Liu, Zhengtao Ding, Senior Member, IEEE
}

\begin{abstract}
Due to the long-distance transmission, external grid cannot effectively support the access point voltage (APV) of the large-scale wind farm (WF), which thus entails DFIGbased WF to provide its ancillary services on reactive power generation. Considering factors of limited down-sized converter capacity, various operating modes of wind turbines (WTs), and the large pressure of large-scale WF communication on the central governor, this paper proposes a distributed active power and reactive power coordination scheme for APV support. A reactive power self-allocation scheme is developed and operated in each WT for reactive power dispatch. Based on the mechanism of distributed consensus control and aligning with the principle of preferential and proportional utilization of spare reactive power capacity (RPC), the proposed method collaborates the rotor side converters and the grid side converters of all WTs but without the necessity of knowing the total RPC. Further, in case that the total RPC is insufficient for the demand, a coordinating factor for RPC extension is generated to weaken the active power. Through a current-enforced PQ coordinating loop, it guarantees a high utilization of converter capacity as well as an exact compensation on the reactive power imbalance, which thereby reduces the wind power curtailment for the RPC release. Several case studies verify the effectiveness of the proposed method.
\end{abstract}

Index Terms-Access point voltage support, active power and reactive power coordination, converter capacity extension, DFIG wind turbine, large-scale wind farm control.

\section{ABBREVIATIONS}

$\begin{array}{ll}\text { APV } & \text { Access Point Voltage } \\ \text { CRS } & \text { Constant Rotor Speed } \\ \text { DFIG } & \text { Doubly Fed Induction Generator } \\ \text { GSC } & \text { Grid Side Converter } \\ \text { MPPT } & \text { Maximum Power Point Tracking } \\ \text { PAC } & \text { Pitch Angle Control } \\ \text { ROC } & \text { Rotor Over-Speeding Control } \\ \text { RP\&AP Reactive Power and Active Power } \\ \text { RPC } & \text { Reactive Power Capactiy } \\ \text { RPD } & \text { Reative Power Demand } \\ \text { RPSA } & \text { Reactive Power Self-Allocation } \\ \text { RSC } & \text { Rotor Side Converter } \\ \text { WF } & \text { Wind Farm } \\ \text { WT } & \text { Wind Turbine }\end{array}$

Manuscript received Aug 20, 2020; revised Nov 18, 2020; accepted Dec 21, 2020.(Corresponding author: Zhengtao Ding).

Z. Dong, Z. Li, Y. Liu and Z. Ding are with the Department of Electrical and Electronic Engineering, the University of Manchester, Manchester, the United Kingdom. E-mail: \{zhen.dong; zhongguo.li; yixing.liu; zhengtao.ding\}@manchester.ac.uk; L. Du is with the Department of Automation, Harbin Institue of Technology, Harbin, China. E-mail: lingyu@ @it.edu.cn

\section{INTRODUCTION}

$\mathbf{W}$ ITH higher penetration of wind energy in power systems, most wind plants nowadays are required to be configured with voltage or reactive power control [1]. Particularly, for large-scale WF locating at remote areas, external grid is not able to provide a satisfactory APV support due to the long-distance transmission [2,3]. Meanwhile, considering the additional construction and maintenance costs needed to install reactive power compensation equipment and the requirements of timely dynamic responses, it is preferable for converterbased WFs to provide ancillary services on reactive power generation with their idle converter capacities $[4,5]$.

The DFIG-based WT, which is equipped with down-sizedesigned RSC and GSC, has been widely applied in wind power generation system due to the favorable performance and economic prospects. Meanwhile, the limited converter capacity of DFIG-based WF entails a dedicated capacity distribution on active power and reactive power for wind power generation as well as APV support. Basically, the active power generated by WTs under a specific wind speed and a specific operating mode occupies a certain amount of converter capacity. Then, the RPC is the sum of the remaining RSC and GSC capacities [6]. If APV support is taken as a priority, when the RPD is larger than the current RPC, part of wind power generation need to be abandoned, and the operating point is driven to move along the converter capacity curve in the direction of decreasing active power until the RPD is met. In this realm, a so-called coordinated active and reactive power control is needed [7, 8] and two points are of particular importance: $i$ ) an accurate curve for description on the maximum converter capacity; $i i$ ) a proper distribution and control scheme on power trajectory migrating alongside the capacity curve for a high converter utilization.

A converter capacity is physically constrained by the permitted rotor and stator current [9]. $P Q$ curve is an widely applied approach for describing the converter capacity, which can be derived from the steady-state phasor model with current constraints enforced. It can serve as the distribution principle, directly determining the active and reactive power setting points, or using the reactive power capacity obtained as the power controller limit $[8,10]$. However, when current constraints are transformed from the current frame to the power frame, inaccuracy possibly occurs due to different levels of model simplifications [11]. As a result, the derived $P Q$ curve cannot match the corresponding current constraints 
well, which means the actual converter capacity may be underestimated (resulting in a lower utilization) or overestimated (resulting in a compromised tracking performance due to the saturation [12]). As an alternative way, putting both modeling and control under the $d q$ frame, excitation current for reactive power can be strictly limited by directly using current constraints (thus realizing a theoretically full converter capacity), whilst the difficulty is that how to bridge the current frame operation with the designed reactive power allocation [13].

From the whole WF point of view, the large amount of dispersed WTs collaborate with each other for RPD support. Accounting for the various wind velocities brought by the different geographical environments, WTs operating in different operating modes take up different converter capacities. In this case, treating all WTs uniformly and using an aggregated model for whole WF description are no longer appropriate $[14,15]$. Accordingly, the conventional uniform reactive power allocation scheme entails that WTs with relatively low RPC encroach too hastily their own active power capacities before the entire RPC of the wind farm is used up, resulting in unnecessary loss of the total active power production. Whereas a desirable dispatch principle is that the entire WF prioritizely uses the existing RPC of both RSC and GSC, allocating reactive power tasks according to the required RPD and RPC of each WT. If it is still insufficient, WTs simultaneously and dynamically reduce active power output to further release RPC.

The large-scale WF with numerous WTs presents a spatially wide-area-distribution characteristics. Centralized structure requires to have large long-distance interactions with the remotely penetrative WTs, which thus brings much more severe communication pressure and the extreme reliance on the central controller [16]. In the other words, centralized control becomes overwhelmed since WTs have been widely dispersed in physical layer while the communication in information layer is still centralized. In contrast, the distributed control has its advancements for such large-scale multi-agent situations, which achieves the consistency between the information layer and the physical layer [17]. In this case, there is only limited-distance communication with adjacent neighbors for cooperation, rather than large amounts of communication with the remote controller, thus avoiding the above problems of centralized framework. Under the distributed framework, [18] proposes a coordination control for WTs participating in system primary control. [14] extracts the kinetic energy for active power regulation. [19] takes both WTs and STATCOMs into consideration for WF voltage control. [20] investigates the voltage control of WF under different frameworks and illustrates the superiority of distributed control over the centralized methods w.r.t. robustness to communication delay, voltage disturbance rejection, etc.

However, the absence of global information due to distributed architecture limits the functional implementation of existing distributed methods, e.g., [14, 18-20] separately consider the active power sharing or reactive power sharing but without taking the coordination problem into consideration. In particular, to date, few papers have mentioned how to implement an autonomous cooperation of all the WTs' available converter capacities for power allocation under the fullrange load. As for the proposed method, apart from retaining merits of distributed framework, the state-of-the-art is that, through a self-determined control of each WT, the systematical cooperative control among WTs and between the RSC and GSC therein is realized simultaneously to meet different levels of RPD. Furthermore, coordination between active and reactive power sharing is achieved with high converter utilization for an effective APV support even under the extremely-heavy load. The contribution can be specified into the following twofolds:

1) A novel RP \& AP coordination strategy is proposed to ensure that each WT is capable of undertaking the assigned RPD beyond its existing RPC. It removes the power loop limit, and updates RPC through the current constraint. Further, employing the difference between reactive power reference and the updated RPC, a cooperating factor is constructed, aiming at dynamically adjusting and exerting the active power when RPD is larger than the original RPC. Through deloading operation, the reduced active power can exactly provide the extra RPC for RPD compensation. The proposed algorithm does not rely on the accuracy of the $P Q$ curve and guarantees a high utilization of the converter capacity during the whole period of RP \& AP coordination.

2) Under the premise of an effective RP \& AP coordination strategy, further, a reactive power self-allocation (RPSA) scheme is developed covering varying levels of RPD. The proposed allocation scheme is realized distributedly in each local controller of WTs rather than the central controller of the WF. Each WT directly determines the individual reactive power setting points according to the RPC of their own RSC and GSC and parameters exchanged with the neighbors, while eliminating the procedures of data collection, computation and reactive power dispatch of WF's central engine. Through parallel computing, the algorithm ensures a proportional RPD sharing, and further, a simultaneous depletion of reactive power and activation of the RP \& AP coordination strategy if RPD further increases, thereby avoiding unnecessary wind curtailment.

The remainder part is arranged as follows. In Section II, a brief introduction on RPC and RPD is given, and thereafter the relationship is analyzed with some principles summarized. Section III presents the proposed method, which includes an overall description on WF architecture, AP \& RP coordination strategy for WT control and RPSA. Case studies and comparison results are provided in IV, followed by the conclusion in Section V.

\section{ANALYSIS ON RPC AND RPD}

\section{A. $R P C$}

The converter capacity of DFIG is determined by RSC and GSC. For RSC, the active and reactive power capacity is constrained by the maximum rotor current $I_{r_{-} \max }$, the maximum stator current $I_{s_{-} \max }$ as well as the maximum rotor voltage. Considering that the rotor speed range is always bounded to 


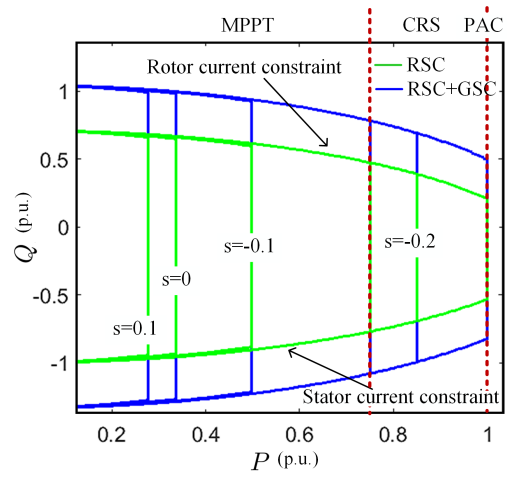

Fig. 1. RPC of DFIG under different operating modes.

avoid conflicting against the maximum rotor voltage, a currentenforced RPC is expressed as [11]:

$Q_{R_{-} \min }=-\left\{U_{s}^{2} I_{s_{-} \max }^{2}-\frac{\left(P+R_{r} \frac{U_{s}^{2}}{X_{m}^{2}}+\left(R_{r}+s R_{s}\right) I_{s_{-} \max }^{2}\right)^{2}}{(1-s)^{2}}\right\}^{\frac{1}{2}}$

$Q_{R_{-} \max }=-U_{s}^{2} / X_{s}+\left\{\frac{\left(X_{m} U_{s} I_{r_{-} \max }\right)^{2}}{X_{s}^{2}}-\frac{P^{2}}{(1-s)^{2}}\right\}^{\frac{1}{2}}$,

where $P$ is the active power; $U_{s}$ is the stator voltage; $R_{s}$ and $R_{r}$ are the stator and the rotor resistance, respectively; $X_{s}$ and $X_{m}$ are the stator and the mutual reactance; $s$ is the slip.

Similarly, the RPC of GSC is

$$
\left\{Q_{G_{-} \max }, Q_{G_{-} \min }\right\}= \pm\left\{S_{G S C}^{2}-s^{2} P^{2} /(s-1)^{2}\right\}^{\frac{1}{2}},
$$

where $S_{G S C}$ is the capacity of GSC, which is usually designed as one third of the DFIG rated capacity for the active power flow generated from the rotor side.

Combining (1)-(2) with (3), the RPC of DFIG under different operating modes can be presented as Fig. 1 shows. The relationship between $P$ and $s$ is established as follows [21]: MPPT mode when $P<0.75$ p.u. (thus $s$ and $P$ is one-toone correspondence following MPPT curve); CRS mode at $s=s_{\text {min }}=-0.2$ when $P \in[0.75,1]$ p.u.; PAC mode for suppressing a constant active power at $P=1.0$ p.u.

\section{B. $R P D$}

Large-scale WF transmits active power to the grid through high-voltage transmission over long distances, which makes APV unable to be compensated through the external grid, so self-compensation from the WF side is encouraged. The RPD to maintain the APV within a proper level can be calculated by [22]:

$$
Q_{D}=-\left\{U_{A P V}^{2} U_{G}^{2} / X_{L}^{2}-P_{w}^{2}\right\}^{\frac{1}{2}}+U_{A P V}^{2} / X_{L},
$$

where $U_{A P V}$ and $U_{G}$ are the voltage of access point and external grid, respectively. $X_{L}$ is the reactance of the transmission line, and $P_{w}$ is the generated wind power of the WF.

RPC of WF and RPD can be depicted in the same figure to illustrate the relationship when using RPC for APV support. As shown in Fig. 2, $Q_{D 1}-Q_{D 3}$ describe different

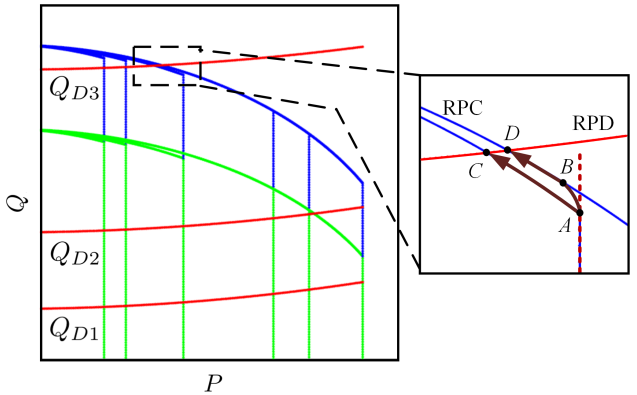

Fig. 2. The relationship between RPC and RPD.

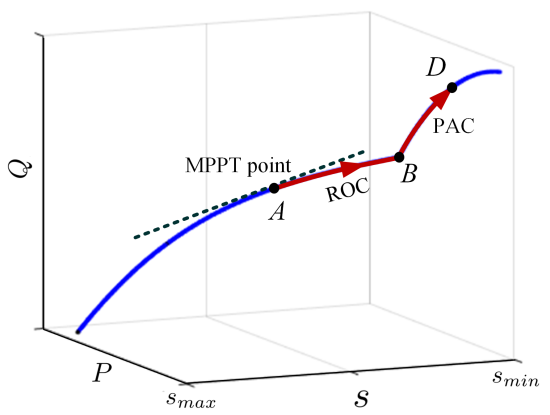

Fig. 3. PQ curve for ROC-prioritized operation under a given wind velocity.

RPD corresponding to different values of APV, of which $U_{A P V 1}<U_{A P V 2}<U_{A P V 3}$. Due to the different operating modes under various wind velocities, the situation can be divided into several cases.

1) RPD within RPC of RSC (Case (i)): As $Q_{D 1}$ shows, it is within the RPC of RSC even when $P=1.0$ p.u. under high wind velocity, indicating that the existing RPD is sufficient enough for the decoupled power control with no interacted actions required.

2) RPD within the sum RPC of RSC and GSC (Case (ii)): If $Q_{D}$ increases from $Q_{D 1}$ to $Q_{D 2}$, RPC of RSC cannot meet the RPD at high wind velocity due to the occupation of the generated active power. Compared with reducing the active power via deloading operation, the participation of GSC is preferred, since $Q_{D 2}$ is still within the sum RPC of RSC and GSC.

3) RPD outside the sum RPC of RSC and GSC (Case (iii)): When $Q_{D}$ is up to $Q_{D 3}$, RPD with high active power generation is outside the RPC and requires an active participation of active power control. Accounting for the operating mode, for $P>0.75$ p.u., since the rotor speed has reached its upper limit, employing PAC is the only choice, and then the operating point will migrate alongside $P Q$ curve corresponding to $s=-0.2$ until reaching the intersection. Whilst for $P<0.75$ p.u., the choice is more flexible since both ROC and PAC can be applied for deloading operation. ROC harvests less wind power with higher rotor speed away from MPPT point, while higher rotor speed can relax the original RPC. As the enlarged part of Fig. 2 shows, assuming that the current operating point is $A$ and point $B$ represents the operating point at the maximum ROC-based deloading 
state, point $A$ will migrate to point $B$ using ROC and then to point $D$ using PAC, or directly migrates to point $C$ if PAC is applied solely. In comparison, $\mathrm{ROC}+\mathrm{PAC}$ is able to meet RPD with less active power curtailment. Further considering more economic benefit (less tear and wear on blade) than PAC, an ROC-prioritized deloading operation is chosen for active power control. Fig. 3 presents the power trajectory for ROC+PAC deloading operation, where mode switch happens at point $B\left(s=s_{\min }\right)$.

Further, if RPD is outside RPC with no intersections, it depicts an extreme situation that RPD cannot be balanced even if all the WF converters are employed for reactive power compensation. Then the system has to rely on other reactive power auxiliary equipment (shunt capacitors, static var compensator, etc.), which is beyond the scope of this paper.

From the above analysis, it can be drawn that, since the combinations of active power and rotor speed corresponding to certain operating modes determine the dynamically changing RPD and RPC, the design of upper-level reactive power dispatch should take into account the pre-defined operating mode sequence and the current status of WTs, to make sure that the lower-level control is able to produce the required reactive power accordingly.

\section{PROposed METHOD}

\section{A. An Overall Description}

The relationship between RPD and the total RPC determines the reactive power allocation and the according AP \& RP coordination scheme, while the total RPC of the entire WF is unknown for the distributed architecture. Moreover, considering the difference and change of wind velocities in both spatial and temporal dimensions, the whole control system needs to formulate the personalized reactive power setting points for each WT according to their current RPC states, as well as the universal AP \& RP coordination and control schemes for single WT with multiple operating modes covering the whole wind velocity range. Based on these considerations, the distributed WF coordination scheme proposed in this paper is as Fig. 4 shows.

The proposed architecture uses a simple communication topology, that is, each WT only communicates with their neighbors. After receiving the external RPD, the WF will send it directly to each WT without any processing, thus saving a powerful and high-cost WF center controller for collecting, processing, and dispatching large amounts of data from and to each WT. RPSA is a self-planed scheme realizing the reactive power allocation based on each WT's current state and limited information obtained from neighbors, as well as meeting the supply and demand balance from the WF point of view. RPSA interacts with the underlying AP \& RP coordination control to obtain capacity information and assign reactive power commands. On the one hand, the bottom layer control needs to ensure that the capacity information transmitted to the upper layer fully squeezes the converter limit. On the other hand, the task of coordination between active power and reactive power is to ensure that the reactive power is capable of effectively following the command sent from the

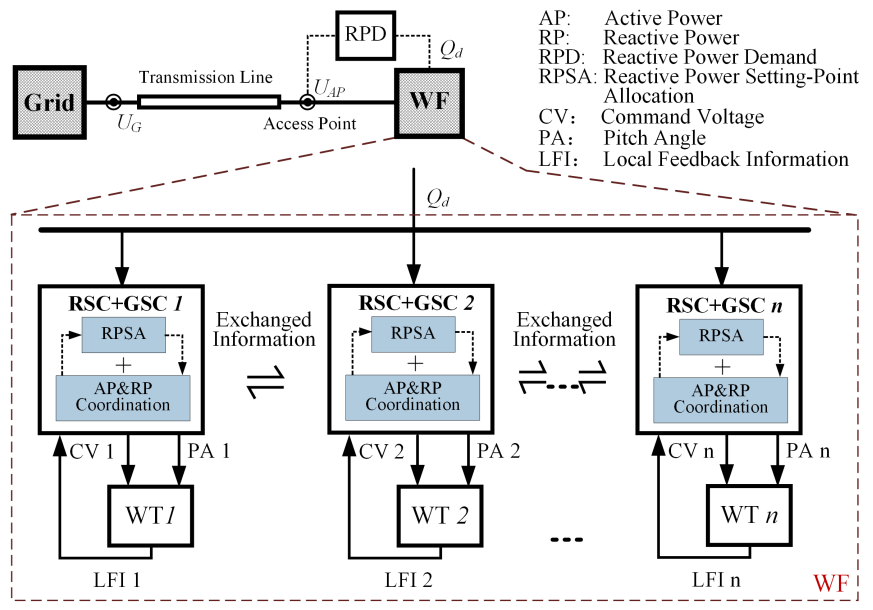

Fig. 4. Control system architecture of grid connected WF.

RPSA all the time. The entire algorithm is applicable to WTs under various working conditions, which can ensure that the AP \& RP coordination control (thus sacrificing active power generation) is not actuated until the existing reactive power of the entire WF is exhausted.

\section{B. AP \& RP Coordination for WT Control}

A $d q$-frame cascaded structure consisting of active and reactive power as outerloop and rotor current as innerloop is commonly applied for lower-level control, whilst $P Q$ curve (1)-(2) can be directly employed in the power loop of RSC as reactive power limits $[8,13]$. Since $P Q$ curve is built by the bridge of rotor current constraint, any inaccuracy between $P$ (or $Q$ ) and $i_{r \max }$ will undermine the reactive power limit fitting the rotor current constraint. On the contrary, from the $d q$ decoupling control perspective, if the limit turns back to the rotor current loop, the RPC of RSC can be expressed as

$$
Q_{R_{-}\{\max ; \min \}}=\frac{3}{2} \omega_{s} \frac{L_{m}}{L_{s}}\left|\boldsymbol{\psi}_{s}\right|\left(i_{r_{-}\{\max ; \min \}}-\frac{\left|\boldsymbol{\psi}_{s}\right|}{L_{m}}\right),
$$

and

$$
\begin{gathered}
i_{r d \_ \text {min }}=\left(-\sqrt{i_{s \max }^{2}-\frac{\left(L_{m} i_{r q}\right)^{2}}{L_{s}^{2}}}+\frac{\left|\boldsymbol{\psi}_{s}\right|}{L_{s}}\right) \frac{L_{s}}{L_{m}}, \\
i_{r d \_\max }=\sqrt{i_{r \max }^{2}-i_{r q}^{2}},
\end{gathered}
$$

where $\psi_{s}$ is stator field; $L_{s}, L_{r}$ and $L_{m}$ are stator selfinductance, rotor self-inductance, and mutual inductance, respectively. In this case, benefiting from the avoidance of the possible inaccurate expression between $P$ and $i_{r \max }$, rotor current constraint is further strictly complied, thereby resulting in a high converter capacity.

Taking Agent $i$ as an example, Fig. 5 shows the control diagram of the proposed scheme. Its function is to ensure that RSC and GSC are able to track the given reactive power references from RPSA, whilst maintaining the full utilization of the converter capacity when AP \& RP coordination is required. Generally, GSC uses $d$-axis current for maintaining 


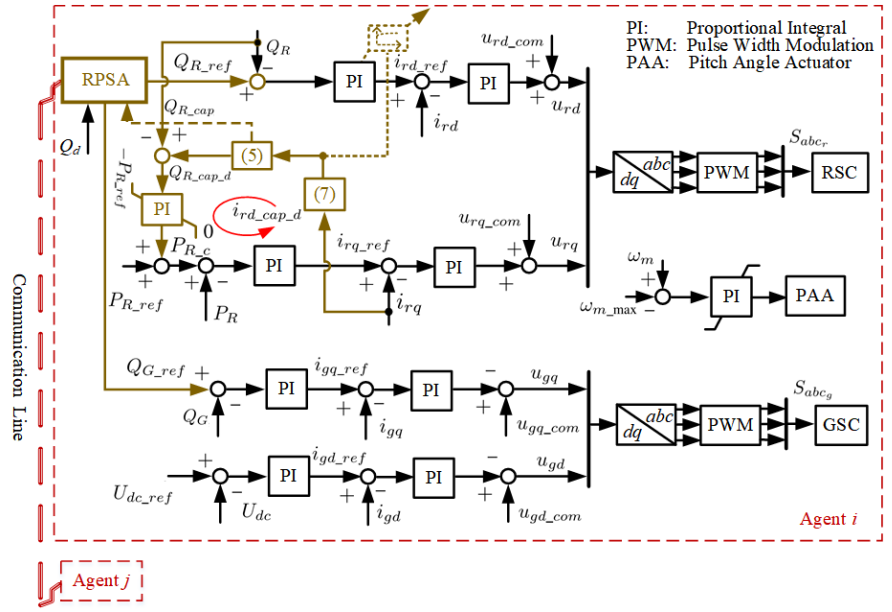

Fig. 5. Control diagram of AP \& RP Coordination scheme.

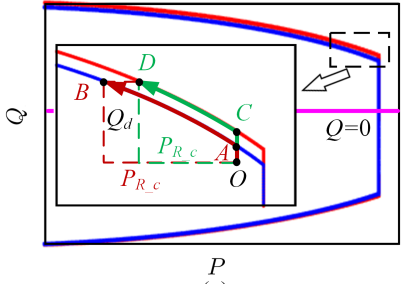

(a)

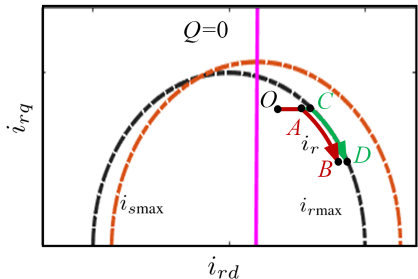

(b)
Fig. 6. Illustration on operating point trajectories under different control constraints. (a) Power trajectories. (b) Rotor current trajectories.

a stable DC-Link voltage while $q$ axis is for reactive power regulation. Besides, pitch angle actuator (PAA) is employed for the balance of the decreased electrical active power and mechanical power. Here, $u_{g d_{-} c o m}=u_{s d}+\omega_{s} L i_{g q}$ and $u_{g q_{-} c o m}=u_{s q}-\omega_{s} L i_{g d}$ are for the purpose of decoupling. The stator-field-oriented control is adopted for RSC control, and $u_{r d_{-} c o m}=-\omega_{r} \sigma L_{r} i_{r q}, u_{r q_{-} c o m}=\omega_{r} \sigma L_{r} i_{r d}+\omega_{r} L_{m}\left|\boldsymbol{\psi}_{s}\right| / L_{s}$ [23]. The limit in reactive power loop is transferred to the current loop and determined by $i_{r q}$ through (7). Then $Q_{R_{-} c a p_{-} d}$ is constructed by $i_{r d_{-} c a p}$ using (5). $P_{R_{-} c}$ is generated by the error of $Q_{R}$ and $Q_{R_{-} c a p_{-} d}$ through a PI controller, of which the upper and lower limits are set as 0 and $-P_{R_{-} r e f}$, respectively. For convenience, the PI controller is called coordinating controller and $P_{R_{-} c}$ is the coordinating factor.

When $Q_{R_{-} r e f}$ is less than the initialized reactive power capacity $Q_{R_{\_} c a p}, P_{R_{-} c}$ will be limited at 0 with no effect on $P_{R_{-} r e f}$. Accordingly, $i_{r d \_r e f}$ will not reach the dynamic limit and $d q$-axis control is fully decoupled. For $Q_{R_{-} r e f}$ larger than $Q_{R_{-} \text {cap }}$, as shown in the enlarged part of Fig. 6 (a), the $P Q$ curve shown in blue is depicted by (1)-(2), while the red curve where points $\mathrm{C}$ and $\mathrm{D}$ are located represents the $P Q$ curve that is strictly determined by the current constraint shown in Fig. 6 (b). We take point $\mathrm{O}$ as the initial operating point, comparing the operating point migration under different $P Q$ curves when facing the same RPD $Q_{d}$. If the blue $P Q$ curve is applied, the power trajectory is O-A-B, while using the red $P Q$ curve is O-A-C-D. From the active power point of view, the extension of $P Q$ curve reduces $\Delta P_{R}$ of the active power curtailment. However, from system control perspective, since the direct expression of $P Q$ curve in red is unavailable, the scheme realization is transferred to the rotor current frame. As Fig. 6 (b) shows, the control objective is to drive the rotor current trajectory to migrate alongside O-A-C-D.

The mechanism is as follows: when the operating point moves to point $\mathrm{C}$ in Fig. 6 (b), $Q_{R}$ cannot track $Q_{R_{-} r e f}$ due to the $d$-axis current limit. Luckily, it is able to trigger the action of coordinating controller once the transient state of $Q_{R}$ goes beyond $Q_{R_{-} c a p_{\_} d}$ and then $i_{r q_{-} r e f}$ is weakened. Since $q$ axis always has enough margin for $i_{r q}$ tracking $i_{r q} r e f$, the decreasing $i_{r q}$ prompts the increase of the dynamic $d$-axis current limit $i_{r d_{-} c a p}$ from point $\mathrm{C}$ to point D in Fig. 6 (b), releasing more $Q_{R}$ through the $d$-axis power and current loop as well as more $Q_{R_{-} \text {cap_d }}$ through the proposed control loop (as the red arrowed loop shows in Fig. 5). This procedure will not end until $Q_{R_{-} c a p_{-} d}=Q_{R}=Q_{R_{-} \text {ref }}$, indicating that the converter capacity released by the reduced active power exactly compensates the remaining RPD.

\section{RPSA}

Depicting the individual states of the $i$ th WT with subscript $i$, the allocation principle of proposed RPSA for WF with $N$ WTs is as follows:

If the total RPC of RSC is larger than RPD $Q_{d}$, only $Q_{R_{-} r e f, i}$ is allocated while $Q_{G_{-} r e f, i}$ is kept as zero. Each WT determines the reactive power reference of RSC according to the proportional principle or fair load-sharing principle[24], which is expressed as

$$
Q_{R_{-} r e f, i}=\frac{Q_{d} Q_{R_{-} c a p, i}}{\sum_{i=1}^{N} Q_{R_{-} c a p, i}}, \quad \text { for } \quad Q_{d} \leq \sum_{i=1}^{N} Q_{R_{-} \text {cap }, i} .
$$

Similarly, we get

$$
\begin{gathered}
Q_{R_{-} r e f, i}=\frac{Q_{d} Q_{R_{-} c a p, i}}{\sum_{i=1}^{N}\left(Q_{R_{-} c a p, i}+Q_{G_{-} c a p, i}\right)}, \\
Q_{G_{-} r e f, i}=\frac{Q_{d} Q_{G_{-} c a p, i}}{\sum_{i=1}^{N}\left(Q_{R_{-} c a p, i}+Q_{G_{-} c a p, i}\right)}, \\
\text { for } \sum_{i=1}^{N} Q_{R_{-} c a p, i}<Q_{d}<\sum_{i=1}^{N}\left(Q_{R_{-} c a p, i}+Q_{G_{-} c a p, i}\right) \\
Q_{R_{-} r e f, i}=\frac{Q_{d}\left(Q_{R_{-} c a p, i}+Q_{G_{-} c a p, i}\right)}{\sum_{i=1}^{N}\left(Q_{R_{-} c a p, i}+Q_{G_{-} c a p, i}\right)}-Q_{G_{-} c a p, i}, \\
Q_{G_{-} r e f, i}=Q_{G_{-} c a p, i}, \\
\text { for } Q_{d} \geqslant \sum_{i=1}^{N}\left(Q_{R_{-} c a p, i}+Q_{G_{-} c a p, i}\right) .
\end{gathered}
$$

Since all the sum items in (8)-(10) are unavailable due to the distributed mechanism, the distributed protocol is developed as 


$$
\begin{aligned}
& k_{l, i}=1 / k_{c, i} \\
& \dot{k}_{c, i}=\alpha\left(\frac{Q_{c a p, i}}{Q_{d} / N}-k_{c, i}\right)-\beta \sum_{j=1}^{N} a_{i j}\left(k_{c, i}-k_{c, j}\right)-z_{i} \\
& \dot{z}_{i}=\alpha \beta \sum_{j=1}^{N} a_{i j}\left(k_{c, i}-k_{c, j}\right),
\end{aligned}
$$

with initial condition $\sum_{i=1}^{N} z_{i}(0)=0 ; a_{i j}$ is determined by the communication topology, see [25, 26] for more information; $k_{c, i}$ and $z_{c, i}$ are parameters exchanged with neighbors; $\alpha$ and $\beta$ are the coefficients responsible for the convergence speed, satisfying $\alpha, \beta>0$.

To prove that the $k_{c, i}$ converges to $\mathbf{1}_{N}^{T} Q_{c a p} / Q_{d}$, it is equivalent to show

$$
e_{i} \triangleq k_{c, i}-\mathbf{1}_{N}^{T} Q_{c a p} / Q_{d}
$$

converges to zero. For the ease of analysis, we introduce a transformation variable $\delta_{i}$ as

$$
\delta_{i}=z_{i}-\alpha \frac{Q_{c a p, i}}{Q_{d} / N}+\alpha \mathbf{1}_{N}^{T} Q_{c a p} / Q_{d} .
$$

It then follows that

$$
\begin{aligned}
& \dot{e}_{i}=-\alpha e_{i}-\beta \sum_{j=1}^{N} a_{i j}\left(e_{i}-e_{j}\right)-\delta_{i} \\
& \dot{\delta}_{i}=\alpha \beta \sum_{j=1}^{N} a_{i j}\left(e_{i}-e_{j}\right) .
\end{aligned}
$$

Let the augmented state variables of the network be $e=$ $\left[e_{1}, \ldots, e_{N}\right]^{T}, \delta=\left[\delta_{1}, \ldots, \delta_{N}\right]^{T}$. Thus, the compact form of (14) can be obtained as

$$
\begin{aligned}
& \dot{e}=-\alpha e-\beta \mathcal{L} e-\delta \\
& \dot{\delta}=\alpha \beta \mathcal{L} e,
\end{aligned}
$$

where $\mathcal{L}$ is the Laplacian matrix associated with the communication graph. Equivalently, we have

$$
\left[\begin{array}{c}
\dot{e} \\
\dot{\delta}
\end{array}\right]=\Psi\left[\begin{array}{l}
e \\
\delta
\end{array}\right]
$$

where $\Psi=\left[\begin{array}{cc}-\alpha I_{N}-\beta \mathcal{L} & -I_{N} \\ \alpha \beta \mathcal{L} & \mathbf{0}_{N}\end{array}\right]$. Therefore, the rest of the proof is to demonstrate that $\Psi$ is stable. Now, we introduce the following transformation

$$
\left[\begin{array}{l}
p \\
q
\end{array}\right]=\mathcal{T}_{1} \mathcal{T}_{2}\left[\begin{array}{l}
e \\
\delta
\end{array}\right]
$$

where

$$
\mathcal{T}_{1}=\left[\begin{array}{cc}
I_{N} & \mathbf{0}_{N} \\
\alpha I_{N} & I_{N}
\end{array}\right], \mathcal{T}_{2}=\left[\begin{array}{cc}
\mathcal{T}_{3}^{T} & \mathbf{0}_{N} \\
\mathbf{0}_{N} & \mathcal{T}_{3}^{T}
\end{array}\right], \mathcal{T}_{3}=\left[\begin{array}{c}
r \\
R
\end{array}\right]_{(18)}^{T}
$$

with $r=\frac{1}{\sqrt{N}} \mathbf{1}_{N}, r^{T} R=0, R^{T} R=I_{N-1}$ and $R R^{T}=$ $I_{N}-r r^{T}$. Let us regroup the variables, $p$ and $q$, by partitioning them as $p=\left[p_{1}, p_{2: N}^{T}\right]^{T}$ and $q=\left[q_{1}, q_{2: N}^{T}\right]^{T}$. Then, we have

$$
\left[\begin{array}{c}
\dot{p}_{1} \\
\dot{q}_{1}
\end{array}\right]=\Psi_{1}\left[\begin{array}{c}
p_{1} \\
q_{1}
\end{array}\right], \quad \Psi_{1}=\left[\begin{array}{cc}
0 & -1 \\
0 & -\alpha
\end{array}\right]
$$

$$
\left[\begin{array}{c}
\dot{p}_{2: N} \\
\dot{q}_{2: N}
\end{array}\right]=\Psi_{2}\left[\begin{array}{c}
p_{2: N} \\
q_{2: N}
\end{array}\right], \Psi_{2}=\left[\begin{array}{cc}
-\beta R^{T} \mathcal{L} R & -I_{N-1} \\
0 & -\alpha I_{N-1}
\end{array}\right]
$$

Note that the eigenvalue of $R^{T} \mathcal{L} R$ are the same as the positive eigenvalues of the Laplacian matrix $\mathcal{L}$. Thus, the eigenvalues of $\Psi_{1}$ are 0 and $-\alpha$, and those of $\Psi_{2}$ are $-\alpha$ with multiplicity $N-1$ and $-\beta \lambda_{i}(\mathcal{L})$ with $\lambda_{i}(L), i=2, \ldots, N$ being the $i$ th smallest eigenvalue of $\mathcal{L}$. Since the null-space spanned by $\Psi$ is the consensus space, i.e., $\left(I_{N},-c I_{N}\right)$, for some constant $c \in \mathbb{R}$, due to the connectivity of the graph, we have $e_{i}$ converges to zero exponentially, and consequently $k_{c, i}$ converges to $\mathbf{1}_{N}^{T} Q_{c a p} / Q_{d}$. Moreover, the exponential convergence rate is given by $\min \left\{\alpha, \beta \lambda_{2}(\mathcal{L})\right\}$. As a result, considering a WF with large numbers of WTs, the convergence speed can be adjustable by tuning $\alpha$ and $\beta$, or by optimizing the communication topology such that the second smallest eigenvalue of $\mathcal{L}$ is maximized.

In (11), $k_{l}$ can act as the reactive power utilization indicator: $k_{l}(\infty)>1$ if $Q_{d}$ is overloaded; $k_{l}(\infty) \leqslant 1$, otherwise. Then, RPSA can be realized by augmenting (11) in the proposed Algorithm 1. The pseudo code of the proposed Algorithm 1 during each dispatch is as follows.

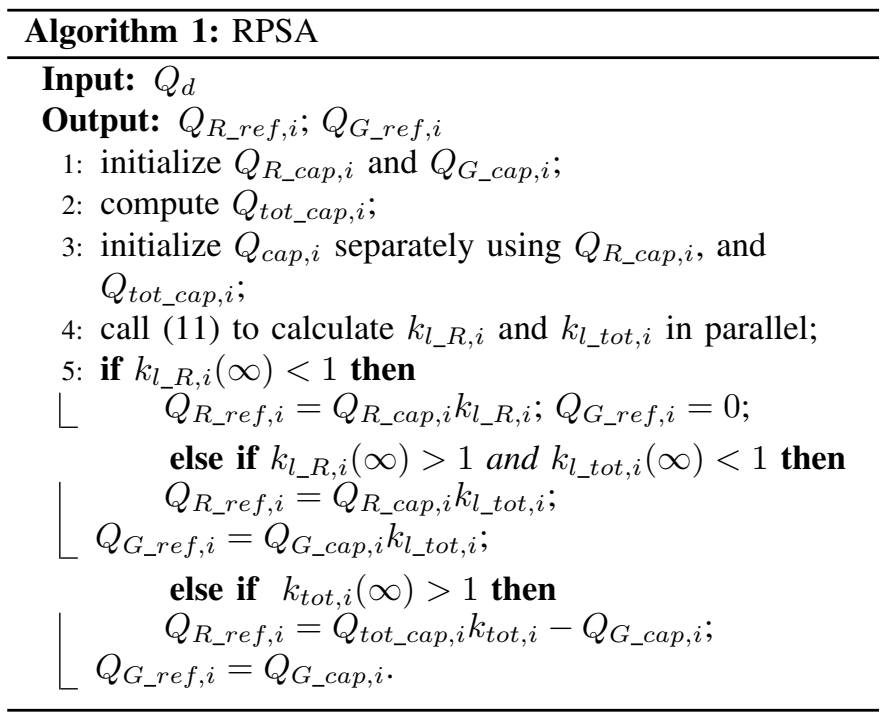

\section{CAse Study}

A 150MW WF composed of $100 * 1.5 \mathrm{MW}$ GE WTs is employed and connected to the external grid through a 200kilometer transmission line $\left(X_{L}=1.65 \times 10^{-3} \mathrm{H} / \mathrm{km}\right)$. WT parameters can be found in [21]. Every $20 \mathrm{WT}$ is regarded as an identical unit sharing the same wind velocity and thus the WF is divided into 5 agents, as shown in Fig. 7. A ring topology is chosen for mutual communication considering simplicity and robustness [27], corresponding to $\mathcal{L}=[2-1$

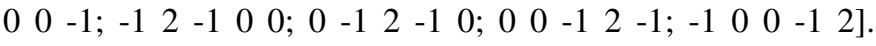
For convenience, in RPSA, the initial state of $k_{c}$ is randomly set around 1 as $[0.8,0.9,1.0,1.1,1.2,1.3]$, and $z$ is set as $\mathbf{0}$. Considering the execution of RPSA and the interval control, the time delay for each dispatch is set as 100ms [28]. Besides, if not specifically marked, unmarked units of the ordinates in all figures below are defaulted as p.u.. 


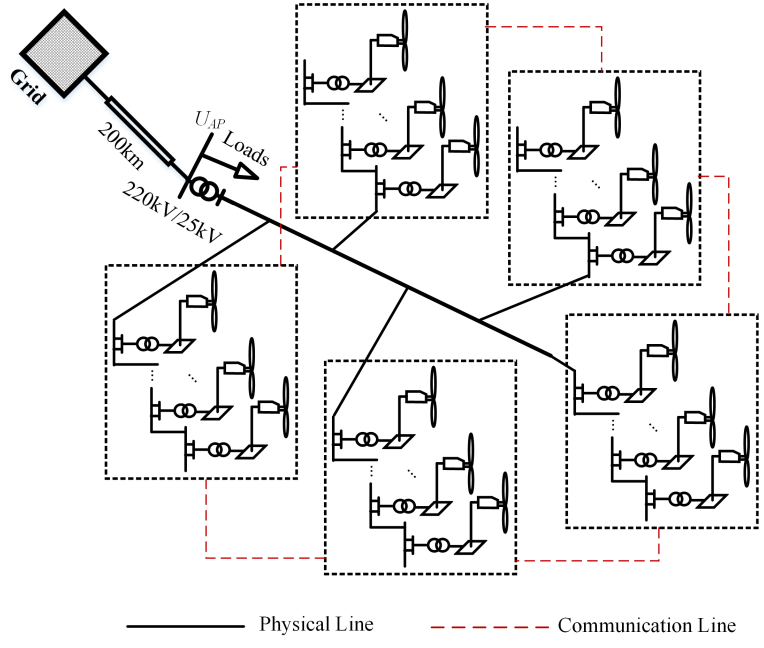

Fig. 7. Configuration of the tested system.
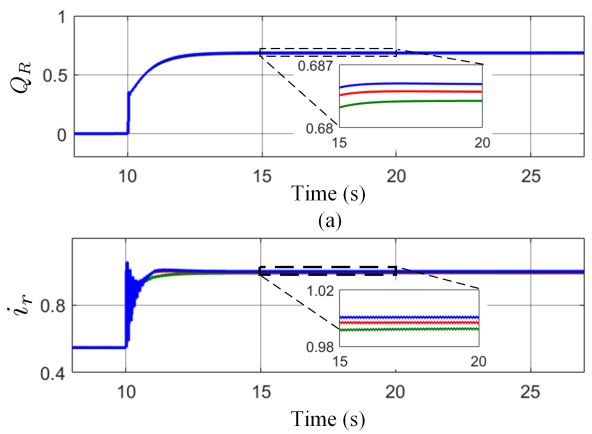

(b)
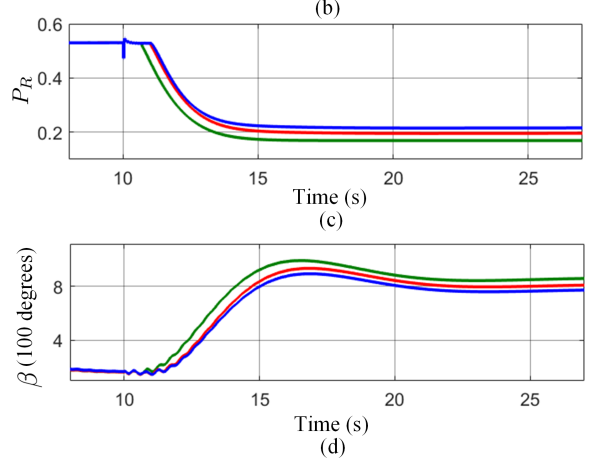

- Method A — Method B — Proposed Method

Fig. 8. Comparison on RSC response using different methods. (a) Reactive power. (b) Rotor current. (c) Active power. (d) Pitch angle.

\section{A. Case 1: Comparison on Utilization of RPC}

In the first step, in order to verify the high utilization of the proposed AP \& RP coordination strategy for RSC, it is compared with other two $P Q$-curve based methods applied in [10] (Method A) and [13] (Method B), where Method A is a fixed RPC determined by assuming a steady-state condition. The capacity is unchanged for a specified $P_{R}$ even for dynamic evaluations, while Method B is derived from circuital expressions and the capacity is dynamically determined using the updated values for $P_{R}$ and $s$. The APV support is solely undertaken by RSC with the reactive power reference of GSC always kept at zero. The wind velocity of the entire wind farm is uniformly set as $11 \mathrm{~m} / \mathrm{s}$. The APV target is set to 1.0 p.u. The system initially operates in active power reference control with $Q_{R}=\mathbf{0}$, and 113.3 Mvar inductive load was suddenly added at $10 \mathrm{~s}$. Fig. 8(a)-(d) respectively presents the responses of RSC reactive power, rotor current, active output and pitch angle. Because RPD exceeds the existing RPC, the three methods enter the AP \& RP coordination stage successively around $11 \mathrm{~s}$, where the proposed method is the latest (the later entry time indicates the higher utilization of RPC). Further, it can be seen that due to the same load on access point, the three methods have very little difference in the performance of reactive power (Fig. 8(a)), and the small difference comes from the RPDs corresponding to the active power at different curtailment levels. However, from the perspective of active power, the proposed method obviously reduces the weakening of active power (Fig. 8(c)). This is because compared with the other two methods, the proposed method has a high rotor current utilization much closer to the limit (Fig. 8(b)), thus broadening the $P Q$ capacity boundary. The extracted converter capacity reduces the active power curtailment, which also corresponds to smaller pitch angles (Fig. 8(d)).

\section{B. Case 2: System Response under Different Loads}

In this case study, the control objective is to maintain $U_{A P}$ at 1.03 p.u. once the APV drops below 0.97 p.u., the wind velocities for each group are set as [13.2 12.0 $10.89 .68 .4] \mathrm{m} / \mathrm{s}$, and the active power references are set according to the MPPT curve as $\left[\begin{array}{lllll}0.93 & 0.72 & 0.53 & 0.37 & 0.25\end{array}\right]$ p.u., respectively. 58.3 Mvar, 91.7 Mvar, 125 Mvar inductive loads are given separately to cover three cases discussed in Section II. The simulation results are shown in Fig. 9. In Case (i), the responses of $k_{l_{-} R}$ and $Q_{R_{-} r e f}$ for dispatch, the output reactive power of RSC and the frequency and voltage responses of APV are presented. The converged value of each $k_{l_{-} R, i}$ is smaller than 1 , indicating that only RSC is needed to be activated for APV support when 58.3 Mvar is given at $1 \mathrm{~s}$. As $Q_{R}$ tracks the self-allocated value $Q_{R_{-} r e f}, U_{A P}$ is stable around 1.03 p.u. In Case (ii), as the load absorbs 91.7 Mvar, $k_{l_{-} R, i}$ converge at 1.314 while $k_{l_{-} t o t, i}$ converge at 0.842 . The participation of GSC eases the burden of RSC and fastens the voltage recovery. In Case (iii), the terminal values of $k_{l_{-} \text {tot }, i}$ are larger than 1 , indicating the active power curtailment is required. GSC remains at the maximum reactive power capability (c), and the remaining tasks are left to RSC. As $Q_{R}$ increases, AP \& RP Coordination is activated at around $5 \mathrm{~s}$.

\section{Case 3: System Response under Wind Velocity Change and Load Change}

Setting the objective value of $U_{A P}$ as 1 p.u., system originally operates at load absorbing 91.7 Mvar. Wind velocity is changed from [13.2 12.010 .89 .68 .4$] \mathrm{m} / \mathrm{s}$ to [13.2, 12.0, 12.0, $10.8,10.8] \mathrm{m} / \mathrm{s}$ at $10 \mathrm{~s}$, and then the load is reduced to 41.7 Mvar at $20 \mathrm{~s}$. Fig. 10 shows the responses of active power $P_{R}$, the coordinating factor $P_{R_{-} c}$, reactive power from RSC $Q_{R}$ and GSC $Q_{G}$, and frequency and voltage of access point. The 


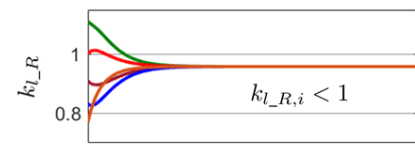

(a) $\quad k_{I_{-} R}$ for dispatch

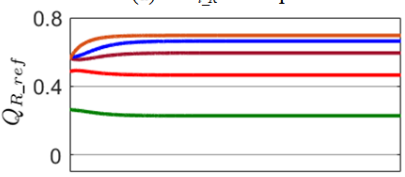

(b) $Q_{R_{-} \text {ref }}$ for dispatch

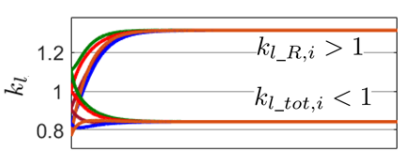

(a) $\quad k_{l_{-} R} k_{l_{-} \text {tot }}$ for dispatch

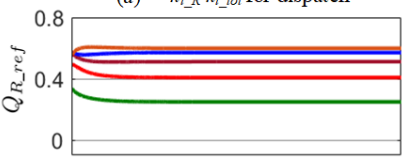

(b) $Q_{R_{-} \text {eff }}$ for dispatch

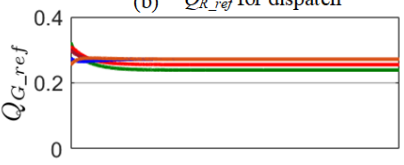

(c) $Q_{G_{-} r e f}$ for dispatch

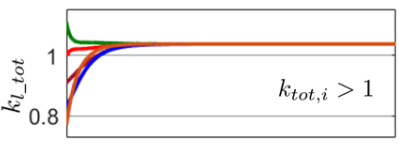

(a) $k_{l_{-} \text {tot }}$ for dispatch

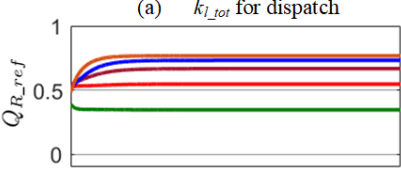

(b) $Q_{R_{-} \text {fef }}$ for dispatch

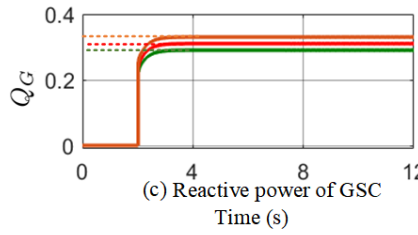

Case (iii)

-wT1 - wT2 - wT3 - wT4 - wT

Fig. 9. System response under different RPD. Case (i) RPD within RPC of RSC. Case (ii) RPD within RPC. Case (iii) RPD outside RPC.

change of wind velocity suddenly increases the active power production (Fig. 10 (a)) and decreases the according RPC. Facing increased RPD and changed RPC, RPSA rearranges the reactive power allocation of each agents, where agents with increased active power (WT3, WT4 and WT5) undertake reduced reactive power tasks (Fig. 10 (c)). Due to the heavily decreased RPC, system migrates from Case (ii) to Case (iii), which can be seen from Fig. 10 (b) and (d). AP \& RP Coordination guarantees a great balance between RPC and RPD, and thus $U_{A P}$ maintains at 1 p.u. with little fluctuation
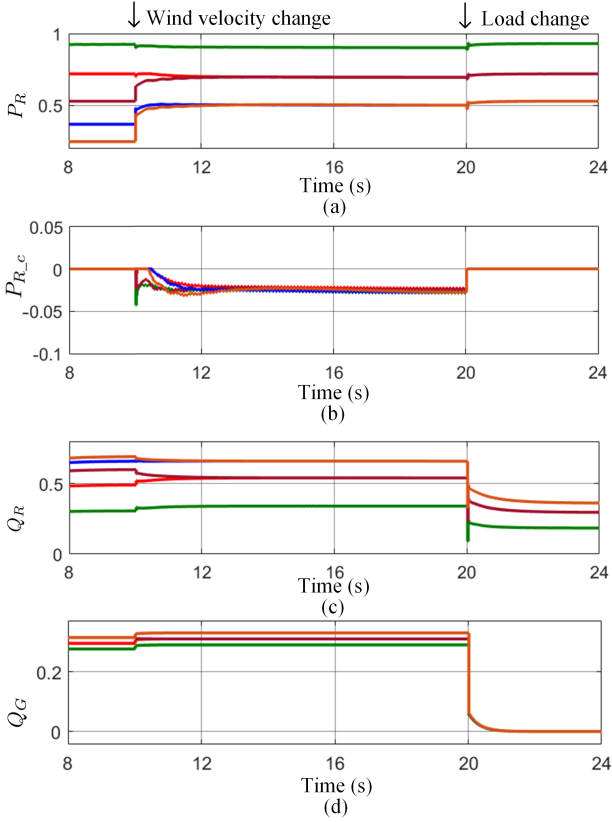

-WT1 —WT2 —WT3-WT4-WT5

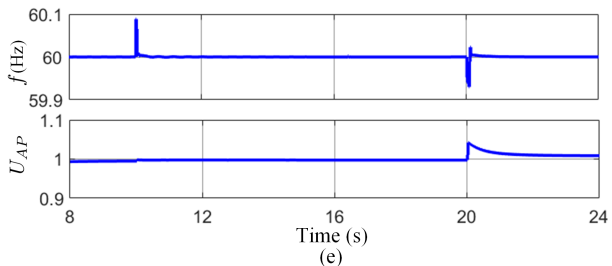

Fig. 10. System response under wind velocity change and load change. (a) Active power. (b) Coordinating factor. (c) Reactive power of RSC. (d) Reactive power of GSC. (e) Access point frequency and voltage.

(Fig. 10 (e)). At $20 \mathrm{~s}$, the sharply reduced RPD drives the system from Case (iii) to Case (i). Since the remaining RPC of RSC is larger than RPD, both $Q_{G}$ and $P_{R_{-} c}$ turn back to zero and the active power generation returns to MPPT mode.

\section{Case 4: Comparison under Communication Line Failure}

A comparison study with other two methods is presented in Fig. 11, where CM, DM and PDM are respectively abbreviated for centralized method, distributed method and the proposed distributed method. For a fair comparison, all of the three methods are based on the fair load-sharing principle. Setting the same condition with Case 3, 91.7 Mvar load is given at 5 s. Communication line disconnection (for $\mathrm{CM}$, it is between WT3 and the central controller, while for DM and PDM, it is between WT2 and WT3.) happens at 8s. Then, wind velocity increases to $[13.2,12.0,12.0,10.8,10.8] \mathrm{m} / \mathrm{s}$ at $10 \mathrm{~s}$. Fig. 11 shows the responses of access point voltage $U_{A P}$, the total reactive power $Q_{R_{-} t o t}$ and total active power $P_{R_{-} t o t}$. It can be seen that in the first $8 \mathrm{~s}$, three methods have the same response. Once line fault happens, WT3 with CM has to stop its service, resulting in a reduction of reactive power as well as active power (Fig. 11(b) and (c)). As the shrunken RPC cannot support the previous RPD, $U_{A P}$ starts to drop Fig. 11(a). While WF with DM or PDM operates normally since WT3 is still connected with the system through the chain communication 

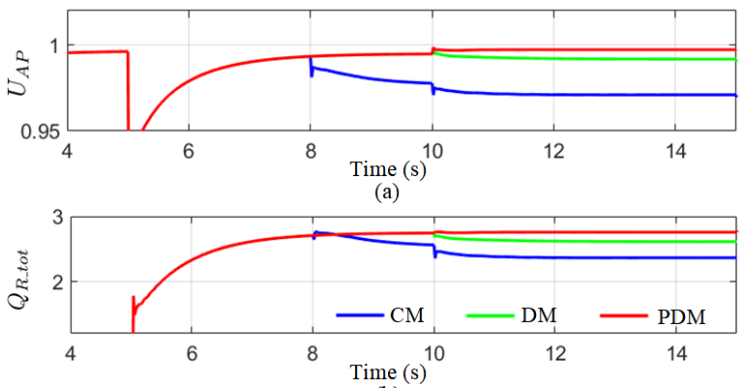

(b)

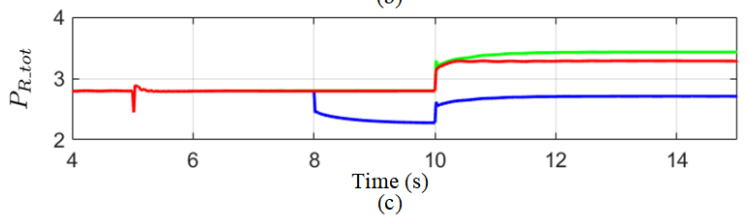

(c)

Fig. 11. WF responses under communication line fault.

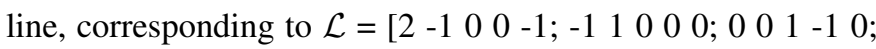

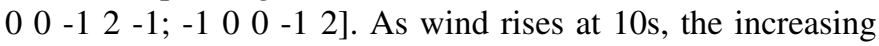
active power crows out the space of reactive power, so the reduced RPC cannot further support the original RPD. $Q_{R_{-} t o t}$ with CM continuously goes down and $Q_{R_{-} t o t}$ with DM begins to drop as well due to the lack of coordination with active power $P_{R_{t} t o t}$, leading to the decrease of $U_{A P}$. In comparison, PDM maintains its APV support through a certain amount of wind curtailment, keeping $U_{A P}$ constant.

\section{E. Case 5: System Response under WT Failure}

With the same wind velocity of Case 2, the system maintains $U_{A P}$ at 1 p.u. when loading 41.7 Mvar. Then, WT5 fails at $15 \mathrm{~s}$ and is removed from the system dispatch. Because of this, the communication topology of WF changes from a loop connection to a chain connection, corresponding to $\mathcal{L}=\left[\begin{array}{lll}1 & -1 & 0\end{array}\right.$ $0 ;-12-10 ; 0-12-1 ; 00-11]$. It can be seen from Fig. 12 that the output of active and reactive power dips to zero, and there is a short-term drop on the frequency of access point, while the active power output of other WTs remains unchanged. Since the increase of other WTs' reactive power output compensates the gap in time, the APV restores to 1 p.u. after a small sag.

\section{CONClusion}

This paper proposes a distributed architecture for the DFIGbased wind farm to provide access point voltage support with less wind power curtailment. Under the unknown relationship between the total reactive power capacity and reactive power demand, an reactive power self-allocation scheme operated in each wind turbine is responsible for the reactive power allocation on rotor side converter and grid side converter, based on the principle of preferential use on spare converter capacity. An active power and reactive power coordination scheme is developed for a heavier reactive power demand. It further extracts the converter capacity through the intervention of the maximum rotor current, and coordinates the distribution of active and reactive power while always maintaining the maximum utilization on converter capacity. The proposed
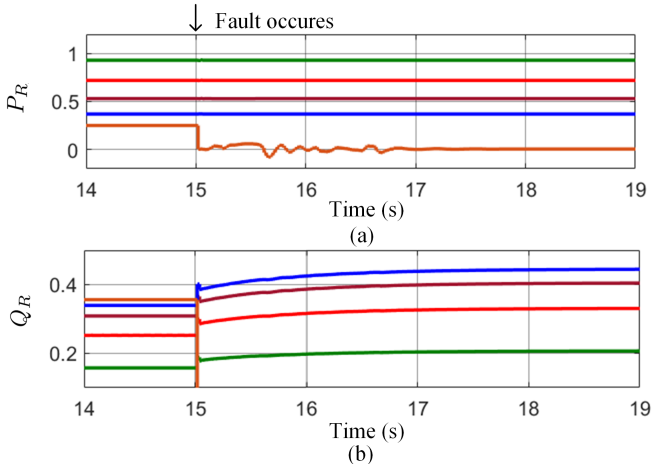

(b)

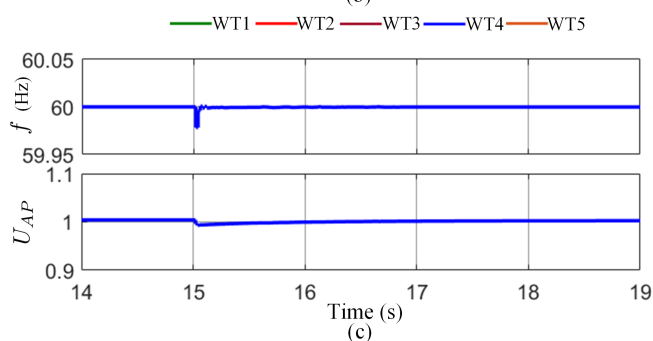

Fig. 12. System response under WT5 failure. (a) Active power. (b) Reactive power of RSC. (c) Access point frequency and voltage.

control scheme ensures the effective support for different levels of reactive power demand and maintains a relatively high wind power yield.

\section{REFERENCES}

[1] H. Sun, Q. Guo, J. Qi, V. Ajjarapu, R. Bravo, J. Chow, Z. Li, R. Moghe, E. Nasr-Azadani, U. Tamrakar, G. N. Taranto, R. Tonkoski, G. Valverde, Q. Wu, and G. Yang, "Review of challenges and research opportunities for voltage control in smart grids," IEEE Trans. Power Syst., vol. 34, no. 4, pp. 2790-2801, 2019.

[2] C. Han, A. Q. Huang, M. E. Baran, S. Bhattacharya, W. Litzenberger, L. Anderson, A. L. Johnson, and A. Edris, "Statcom impact study on the integration of a large wind farm into a weak loop power system," IEEE Trans. Energy Conversion, vol. 23, no. 1, pp. 226-233, 2008.

[3] Q. Guo, H. Sun, B. Wang, B. Zhang, W. Wu, and L. Tang, "Hierarchical automatic voltage control for integration of large-scale wind power: Design and implementation," Elect. Power Syst. Res., vol. 120, pp. 234 241, 2015.

[4] R. Yousefian, R. Bhattarai, and S. Kamalasadan, "Transient stability enhancement of power grid with integrated wide area control of wind farms and synchronous generators," IEEE Trans. Power Syst., vol. 32, no. 6 , pp. 4818-4831, 2017.

[5] Y. Lu and K. Tomsovic, "Wide area hierarchical voltage control to improve security margin for systems with high wind penetration," IEEE Trans. Power Syst., vol. 33, no. 6, pp. 6218-6228, 2018.

[6] M. Kayikci and J. V. Milanovic, "Reactive power control strategies for dfig-based plants," IEEE Trans. Energy Conversion, vol. 22, no. 2, pp. 389-396, 2007.

[7] H. Zhao, Q. Wu, J. Wang, Z. Liu, M. Shahidehpour, and Y. Xue, "Combined active and reactive power control of wind farms based on model predictive control," IEEE Trans. Energy Conversion, vol. 32, no. 3, pp. 1177-1187, 2017.

[8] S. Ghosh, Y. J. Isbeih, R. Bhattarai, M. S. E. Moursi, E. F. El-Saadany, and S. Kamalasadan, "A dynamic coordination control architecture for reactive power capability enhancement of the dfig-based wind power generation," IEEE Trans. Power Syst., vol. 35, no. 4, pp. 3051-3064, 2020.

[9] R. R. Londero, C. d. M. Affonso, and J. P. A. Vieira, "Long-term voltage stability analysis of variable speed wind generators," IEEE Trans. Power Syst., vol. 30, no. 1, pp. 439-447, 2015.

[10] R. J. Konopinski, P. Vijayan, and V. Ajjarapu, "Extended reactive capability of dfig wind parks for enhanced system performance," IEEE Trans. Power Syst., vol. 24, no. 3, pp. 1346-1355, 2009. 
[11] H. Pulgar-Painemal, "Enforcement of current limits in dfig-based wind turbine dynamic models through capability curve," IEEE Trans. Sustain. Energy, vol. 10, no. 1, pp. 318-320, 2019.

[12] Z. Dong, Z. Ding, B. Wang, and D. Xu, "Optimized anti-windup coordinating strategy for torque-maximized high-speed flux-weakening operation of constrained induction motor drives," IEEE J. Emerging Sel. Top. Power Electron., DOI 10.1109/JESTPE.2020.2985218.

[13] J. Ouyang, T. Tang, J. Yao, and M. Li, "Active voltage control for dfig-based wind farm integrated power system by coordinating active and reactive powers under wind speed variations," IEEE Trans. Energy Conversion, vol. 34, no. 3, pp. 1504-1511, 2019.

[14] X. Gao, K. Meng, Z. Y. Dong, D. Wang, M. S. El Moursi, and K. P. Wong, "Cooperation-driven distributed control scheme for large-scale wind farm active power regulation," IEEE Trans. Energy Conversion, vol. 32, no. 3, pp. 1240-1250, Sep. 2017.

[15] P. Mahish and A. K. Pradhan, "Distributed synchronized control in grid integrated wind farms to improve primary frequency regulation," IEEE Trans. Power Syst., vol. 35, no. 1, pp. 362-373, 2020.

[16] W. Meng, X. Wang, and S. Liu, "Distributed load sharing of an inverterbased microgrid with reduced communication," IEEE Trans. Smart Grid, vol. 9, no. 2, pp. 1354-1364, 2018.

[17] Z. Dong, Z. Li, Y. Dong, S. Jiang, and Z. Ding, "Fully-distributed deloading operation of dfig-based wind farm for load sharing," IEEE Trans. Sustain. Energy, pp. 1-1, 2020.

[18] Z. Wang and W. Wu, "Coordinated control method for dfig-based wind farm to provide primary frequency regulation service," IEEE Trans. Power Syst., vol. 33, no. 3, pp. 2644-2659, 2018.

[19] Y. Guo, H. Gao, and Q. Wu, "Distributed cooperative voltage control of wind farms based on consensus protocol," Int. J. Electr. Power Energy Syst., vol. 104, no. JAN., pp. 593-602, 2019.

[20] S. Asadollah, R. Zhu, and M. Liserre, "Analysis of voltage control strategies for wind farms," IEEE Trans. Sustain. Energy, vol. 11, no. 2, pp. 1002-1012, 2020.

[21] N. W. Miller, J. J. Sanchez-Gasca, W. W. Price, and R. W. Delmerico, "Dynamic modeling of ge 1.5 and $3.6 \mathrm{mw}$ wind turbine-generators for stability simulations," in 2003 IEEE Power Eng. Soc. Gen. Meet., vol. 3, pp. 1977-1983 Vol. 3, Jul. 2003.

[22] M. Zhou, J. Ge, F. Guo et al., "Voltage and reactive power emergency control strategy of wind farm cluster against cascading trip-off," Autom. Electr. Power Syst., vol. 40, no. 5, pp. 71-77, 2016.

[23] H. Abu-Rub, M. Malinowski, and K. Al-Haddad, Power electronics for renewable energy systems, transportation and industrial applications. John Wiley \& Sons, 2014.

[24] W. Zhang, Y. Xu, W. Liu, F. Ferrese, and L. Liu, "Fully distributed coordination of multiple dfigs in a microgrid for load sharing," IEEE Trans. Smart Grid, vol. 4, no. 2, pp. 806-815, Jun. 2013.

[25] S. S. Kia, J. Cortés, and S. Martinez, "Dynamic average consensus under limited control authority and privacy requirements," Int. J. Robust Nonlin. Contr., vol. 25, no. 13, pp. 1941-1966, 2015.

[26] Z. Ding, "Consensus control of a class of lipschitz nonlinear systems," Int. J. Contr., vol. 87, no. 11, pp. 2372-2382, 2014.

[27] S. Bolognani, R. Carli, G. Cavraro, and S. Zampieri, "On the need for communication for voltage regulation of power distribution grids," IEEE Trans. Contr. Netw. Syst., vol. 6, no. 3, pp. 1111-1123, 2019.

[28] B. A. Robbins, C. N. Hadjicostis, and A. D. DomÃnguez-GarcÃa, "A two-stage distributed architecture for voltage control in power distribution systems," IEEE Trans. Power Syst., vol. 28, no. 2, pp. 1470-1482, 2013.

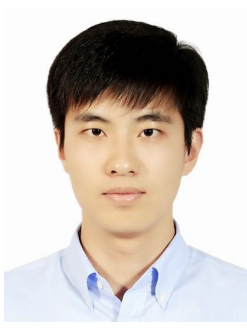

Zhen Dong (S'18) received the B.Eng. degree and M.Eng. degree in Electrical Engineering from Harbin Institute of Technology (HIT), Harbin, China, in 2016 and 2018, respectively. He is currently working toward the Ph.D. degree in Control Systems in the Department of Electrical and Electronic Engineering, The University of Manchester, Manchester, U.K.. His research interests include high performance motor control, and distributed management of grid-connected wind farm.

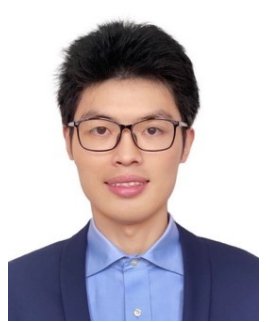

Zhongguo Li (S'20) received the first B.Eng. degree in electrical and electronic engineering from the University of Manchester, Manchester, UK, and the second B.Eng. degree in communication engineering from Jilin University, Jilin, China, in 2017. He is currently a Ph.D. candidate in electrical and electronic engineering with the University of Manchester. From November 2020, he has been a Research Associate with the Department of Aeronautical and Automotive Engineering, Loughborough University, Loughborough, U.K. His research interests include distributed optimization, game theory, decision-making and their applications.

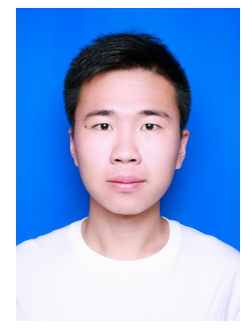

Lingyu Du received the B.S. degree in Automation from Harbin Institute of Technology (HIT), Harbin, China, in 2019. He is currently working toward the M.S. degree in the Department of Control Science and Engineering at HIT. His research interests include model predictive control and model-based reinforcement learning.

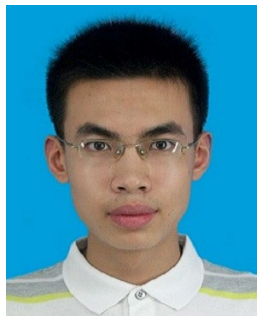

Yixing Liu received the B.E. (Hons.) degree in electrical and electronic engineering from the University of Manchester, Manchester, U.K., and North China Electric Power University, Beijing, China, in 2019. He is currently pursuing the Ph.D. degree in electrical and electronic engineering, University of Manchester, Manchester, U.K

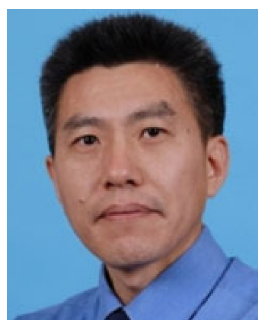

Zhengtao Ding (SM'03) received the B.Eng. degree from Tsinghua University, Beijing, China, in 1984, and the M.Sc. degree in systems and control, and the Ph.D. degree in control systems from the University of Manchester Institute of Science and Technology, Manchester, U.K. in 1986 and 1989, respectively. After working as a Lecturer with Ngee Ann Polytechnic, Singapore, for ten years, he joined the University of Manchester in 2003, where he is currently Professor of Control Systems with the Dept of Electrical and Electronic Engineering. He is the author of the book: Nonlinear and Adaptive Control Systems (IET, 2013) and has published over 260 research articles. His research interests include nonlinear and adaptive control theory and their applications, more recently network-based control, distributed optimization and distributed machine learning, with applications to power systems and robotics. Prof. Ding has served as an Associate Editor for the IEEE Transactions on Automatic Control, IEEE Control Systems Letters, and several other journals. He is a member of IEEE Technical Committee on Nonlinear Systems and Control, IEEE Technical Committee on Intelligent Control, and IFAC Technical Committee on Adaptive and Learning Systems. 